\title{
Artificial neural mitosis
}

\section{Introduction}

Natural biologic organisms are much more complex than automatic and electronic devices. Nevertheless, there are some operations and behaviors in the organization of the biologic beings that might be useful for the human intuition. The experience shows some inspirations arisen from physiological systems, mainly the system of human beings. Man has had as inspiration his/her own central nervous system, which is the most complicated system of all and has been source of different biological inspirations, creating the assembly of some devices, such as the case of the artificial neural networks. These devices sometimes have some limitations in their design and operation when intending to provide a solution to a particular problem. However, these systems are still in a transformation process; that is, the development of the networks is still open to changes. In other words, new paradigms must be developed, such as learning and the massive parallelism of the neural networks.

The synthesis of the biologic neurons is important and difficult, because it is from it that the human brain originates. The brain is an inspiration source to generate the electronic devices or artificial neural network that constitutes the synthesis of the artificial neurons. Artificial neural networks are characterized by their autonomy and logic flexibility; these execute at least one part of the functions of the central nervous system. ${ }^{1}$

\section{Neural mitosis as an extension of the theoretical frame}

Neural mitosis or neuron multiplication: olfactory, auditory, visual, taste, constitute new paradigms with excellent benefits to the modern medicine and neural physiology. Neurons are considered to be automatic physiological devices known as a "black box" that react to stimuli and respond as independent functional units. Biologic neurons have clearly defined characteristics; that is, these units receive signals that can be excitatory or inhibitory. In order for a biologic neuron to emit it is necessary that it receives excitatory stimuli. After a determined time, the neuron will emit "only one" signal.

The central nervous system (CNS) is very complex. Consequently, for its analysis the biologic neuron is considered as an independent unit. In the first phase of the problem, the structure and functioning of the processing units must be isolated. The objective of the second phase is to understand how the neurons are organized and how they relate in between, considering for the analysis the base of these elements of individual information processing. It is known that the artificial neuron is a huge simplification of the real functioning of a biologic neuron. However, the creation of an unreal artificial neural that goes beyond the limits is proposed. For this, the daughter neurons are generated from the mother neuron with the same DNA. Thus, the DNA of the mother neuron and the daughter neurons will have the following relation: 1) They will have the same input, coming from the neuron corresponding to the previous layer, 2) They will share the same tendency vector and, 3) They will have the same transfer function. The mother neuron and the daughters, as constituent units of an Artificial Neural Network (ANN) only differentiate with their connection weight and output signal. In this scenario of inspiration,
Volume I Issue I - 2017

\author{
Lácides Pinto Mindiola, Olenka Gómez Julio \\ University of La Guajira, Colombia
}

Correspondence: Lácides Pinto Mindiola, University of La Guajira, Riohacha, Colombia, Email Ipintom@uniguajira.edu.com

Received: March 22, 2017| Published: April 21, 2017

the neuronal functioning that incorporates the concept of neuronal multiplication is taken from granted, similar to the phenomenon of cellular mitosis (phenomenon that happens between the cells of the intestinal epithelium and the tissues of the epidermis). Therefore, the artificial neural mitosis is proposed as a new paradigm attributable to artificial neurons; this event can definitely be object of a deep and complex reflection in the specific area of biologic neurons.

Artificial neural mitosis is a process of equal distribution of the hereditary material, DNA. Figure 1 shows different version of what could be the "mitosis of an artificial neuron". Only after the phenomenon of the neural mitosis, the daughter neurons might produce demand of necessary signals along to the signal attributable to the mother neuron. ${ }^{2}$

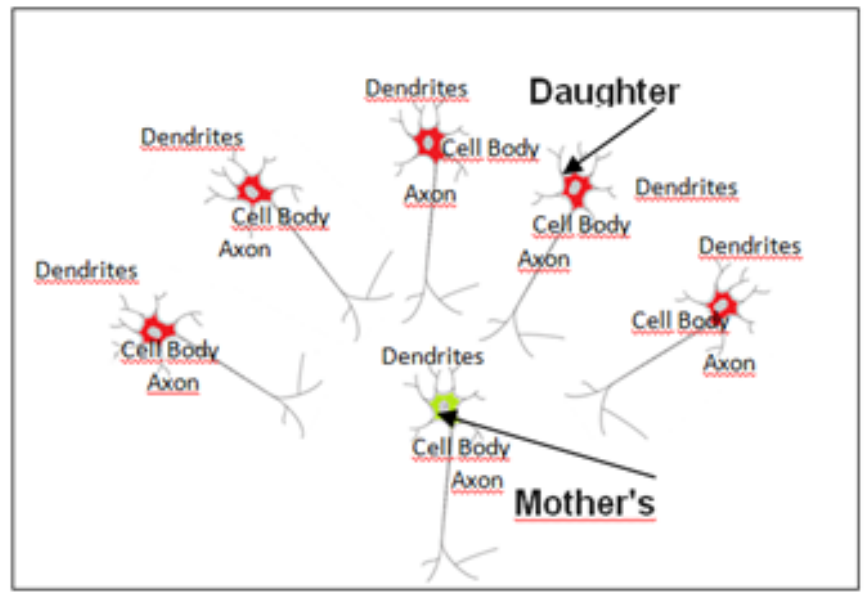

Figure I Schematic drawing of artificial neural mitosis.

\section{Conclusion}

Cellular mitosis typical of the tissues of the intestinal epithelium and the epidermis is a source of biological inspiration; this mitosis was the origin of "artificial neural mitosis" and offers the idea of the possible "biological neural mitosis".

\section{Acknowledgements}

None.

\section{Conflict of interest}

The author declares no conflict of interest. 


\section{References}

1. Biological foundations-neuron communication
2. Newman JR. El Mundo de las matemáticas. Printer Industria Gráfica, Spain: SA Barcelona España; 1983. 